\title{
16. Applications of the theory of pseudo-regular functions to the type-problem of Riemann surfaces,*
}

\author{
By Shizuo KakUTanI
}

(Received May 4, 1936)

The object of this paper is to apply the theory of pseudo-regular functions to the type-problem of Riemann surfaces of meromorphic functions.

Pseudo-regular functions (or pseudo-conformal transformations) were first studied by H. Grötzsch $\left({ }^{1}\right)$, who proved that Picard's theorem can be generalized to a class of pseudo-regular transformations ("Abbildung von beschränkter infinitesimaler Verzerrung").

Recently M. Lavrentieff $\left({ }^{2}\right)$ proved the same theorem for some wider class of functions.

In $\$ 1$ we define pseudo-regular functious and pseudo-conformal transformations and give some properties of these functions.

Then the generalization of Picard's theorem to some class of these functions is established with the aid of Ahlfors' $\left({ }^{3}\right)$ method, the result being the same with that obtained by M. Lavrentieff $\left({ }^{4}\right)$.

This theorem is applied $\left({ }^{5}\right)$ in $\$ 2$ to the problem of the type of simply connected infinitely many sheeted open Riemann surface $\sum$, and a method to determine the type of $\sum$ is obtained.

From this we obtain some results on the properties of Riemann surfaces of meromorphic functions, which are already studied by $A$. Speiser and L. Ahlfors $\left({ }^{6}\right)$.

* The result of this paper was presented to the Meeting of Physico-Mathematical society of Japan on 28. Sept. 1935 (Osaka).

(1) H. Grötzsch: Über eine Verzerrung bei schlichten nicht konformen Abbildung und iber eine damit zusammenhängende Erweiterung des Picardschen Satzes, Leipziger Berichte, 80 (1928).

() M. Larrentieff: Sur une class de repésentations continues, C. R. 200 (1935).

${ }^{3}$ ) L. Ahlfors: Untersuchungen zur Theorie der konformen Abbildung und der ganzen Funktionen, Acta Soc. Scient. Fennicae, Nova Series A. 1 (1930).

( $\left.{ }^{4}\right)$ M. Lavrentieff: loc. cit. ( $\left.{ }^{2}\right)$.

$\left.{ }^{5}\right)$ In this application we use Picard's theorem only in the form which was given by H. Grötzsch.

$\left({ }^{6}\right)$ (a) A. Speiser: Probleme aus dem Gebiet der ganzen transzendenten Funktionen, Comment. Math. Helv., 1 (1929).

(b) L. Ahlfors: Sur les domaines dans lesquels une fonction méromorphe prend des valeurs appartenant à une région donnée, Acta Soc. Scient. Fennicae, Nova Ser. A. 2 (1933).

(c) L. Ahlfors: Quelques propriétés des surfaces de Riemann correspondant aux fonctions méromorphes, Bull. de la soc. math. France, 60 (1932). see also: 
In $\$ 3$ we deal with Spoiser's Problem('), although this method can be applied to a much wider class of problems, and a sufficient condition that $\sum$ is hyperbolic is obtained, by which we can show the impossibility of determining the type of Riemann surface $\sum$ only from the order of increment of the function $\mu(\theta)$ (or $n(\theta))\left({ }^{8}\right)$ of its topological tree; for it is possible to construct two Riemann surfaces $\Sigma_{1}$ and $\sum_{2}$, the one being parabolic and the other hyperbolic, and yet for which the inequality $\mu_{1}(\theta) \geqq \mu_{2}(\theta)$ holds for all $\theta \geqq 0, \mu_{1}(\theta)$ and $\mu_{2}(\theta)$ denoting the "tree-functions" of topological trees of $\sum_{1}$ and $\sum_{2}$ respectively.

I wish to express my hearty thanks to Profs. T. Shimizu and K. Yosida for their kind advices in the course of this work. Theorems in $\$ 1$ are obtained by $\mathrm{K}$. Yosida.

\section{$\S 1$.}

A uniform continuous function

$$
w=f(z)=u(x, y)+i v(x, y), \quad z=x+i y,
$$

is callcd pseudo-regular in a domain $D$, if it satisfies the following conditions:

1. $u_{x x}, u_{y}, v_{x}, v_{y}$ exist and are continuous in $D$.

2.

$$
J(x, y) \equiv\left|\begin{array}{cc}
u_{x} & u_{y} \\
v_{x} & v_{y}
\end{array}\right|>0
$$

except possibly at most the countable set of points $z=x+i y$ wherc $J(x, y)$ $=0$, which, however, has no point of accumulation inside of $D$.

3. At the point $z_{0}$ where $J(x, y)=0$, a sufficiently small neighbourhood of $z_{0}$ is transformed topologically on the piece of Riemann surface which has its only one algebraic singularity of order $n-1\left({ }^{9}\right)$ at $u_{0}=u\left(x_{0}, y_{0}\right)+i v\left(x_{0}, y_{0}\right)$.

(d) L. Ahlfors: Über die knnforme Abbildung, wn Überlagerungsfächen, Attonde Skandinaviska Matematikerkongressen, Stockho!m 1934, and

(e) I. Ahlfors: Zur Theorie der Oberlagerungsflächen, Acta Nath., 65 (1935).

( 7 ) (a) A. Speiser, loc. cit., (6) (a) see also:

(b) A. Speiser: Über beschränkte automorphe Funktionen, Comment. Math. Helv.,

(c) Z. Kobayashi: On the type of Riemann surfaces, Science Reports of the Tokyo Bunrika Daigaku, Section A. 2. (1935) No. 42.

( 3$)$ as for $\mu(\theta)$ see:

(a) Z. Kohafashi: Theorems on the conformal representation of Riemann surfaces, Science Reports of the Tokyo Bunrika Daigaku, Section A, 2 (1935), No. 39. as for $n(\theta)$ see :

(b) R. Nevanlinna: Ein Satz ïber die konforme Abbildung Riemannscher Flächen, Comment. Math. Helr., 5 (1938). see also:

(c) L. Ahlfors: Zur Bestimmung des Typus einer Riemannschen Fläche, Comment. Math. Helv., 3 (1931).

( $\left.{ }^{9}\right) \quad n$ is an integer greater than 1 . It means that $n$ sheets of surface meet in one point $z_{0} . \quad n$ is called "nultiplicity" of $u_{0}$-point $z=\hat{n}_{0}$. 
These conditions mean that this function considered as a transformation (or representation) is topologically equivalent to a regular function.

We say that $f(z)$ is preudo-regular at $z_{0}$ if it is pseudo-regular in some neighbourhood of $z_{0}$.

If we put as usual

$$
\begin{aligned}
& E=u_{x}^{2}+v_{x}^{2}, \\
& F=u_{x} u_{y}+v_{x} v_{y}, \\
& G=u_{y}^{2}+v_{y}^{2}
\end{aligned}
$$

and

$$
p_{r}(z) \equiv p(z) \equiv p(x, y)=\frac{E+G}{J} \equiv \frac{E+G}{\sqrt{E G}-F^{2}},
$$

then

$$
p(x, y) \geqq 2\left({ }^{10}\right) \text {. }
$$

We shall first explain the meaning of $p(x, y)$. At the point where $J(P) \neq 0$, an infinitesimal circle $C$ with centre $P$ is transformed by this function into an infinitesimal ellipse $E$ with centre $f(P)$ (if we neglect infinitesimals of order higher than 1 ).

Assuming, without loss of generality, $P=0, f(P)=0$, a circle $z=r e^{i_{\theta}}$ (or $x=r \cos \theta, y=r \sin \theta$ ) is transformed into an ellipse

$$
\begin{aligned}
& u=r\left(u_{x}(0,0) \cos \theta+u_{y}(0,0) \sin \theta\right), \\
& v=r\left(v_{x}(0,0) \cos \theta+v_{y}(0,0) \sin \theta\right),
\end{aligned}
$$

whose major and minor axes are of lengths respectively

$$
r_{1}=\sqrt{\frac{E+G}{2}+\sqrt{\left(\frac{E+G}{2}\right)^{2}-J^{2}}}
$$

and

$$
r=\sqrt{\frac{E+G}{2}-\sqrt{\left(\frac{E+G}{2}\right)^{2}-J^{2}} .}
$$

If we put

$$
q_{f}(z) \equiv q(z) \equiv q(x, y)=\frac{r_{1}}{r_{2}}(\geqq 1)
$$

then

$$
p(x, y)=q(x, y)+\frac{1}{q(x, y)} .
$$

Thus $p(x, y)$ and $q(x, y)$ denote the magnitude of the ratio of major and minor axes, or the degree of deformation "im kleinen" (Verzerrungsgrad im kleinen), and as $p(x, y)$ becomes small (that is, when it approaches to 2), the transformation tends to the conformal one.

(10) Equality holds when and only when $u_{x}=v_{y}, u_{y}=-v_{x}$, so that, if $p(x, y) \equiv 2$ in $D$ then $f(z)$ is regular inside of $D$. 
Since $r \sqrt{E}$ and $r \sqrt{G}$ are the lengths of the radii of $E$ corresponding to the radii of $C$ on $x$-axis and $y$-axis respectively, we have

$$
r_{1} \geqq r \sqrt{E} \geqq r^{2}, \quad r_{1} \geqq r \sqrt{G} \geqq r_{2}
$$

or

$$
\frac{r_{1}^{2}}{r^{2}} \geqq E \geqq \frac{r_{3}^{2}}{r^{2}}, \quad \frac{r_{1}^{2}}{r^{2}} \geqq G \geqq \frac{r_{2}^{2}}{r^{2}} .
$$

On the other hand, as is easily seen by (1) and (2),

$$
r^{2} \cdot J=r_{1} \cdot r_{2} .
$$

This, combined with (3), gives

$$
q \cdot J \geqq E \geqq \frac{J}{q}, \quad q \cdot J \geqq G \geqq \frac{J}{q} .
$$

Now we shall study the properties of pseudo-regular functions.

At first we give three theorems without proof, as these are almost evident from the definitions of $p(z)$ and $q(z)$.

Theorem 1. If $w=f(z)$ is psendo-regular and $\zeta=g(w)$ is regular, then

$$
\zeta=g(f(z)) \equiv h(z)
$$

is a pseudo-regular function, which has the same $p(z)$ and $q(z)$ with $f(z)$.

Theorem 2. If $w=f(z)$ is regular and $\zeta=g(w)$ is pseudo-regular, then

$$
\zeta=g(f(z)) \equiv h(z)
$$

is a pseudo-regular function, whose $p(z)$ and $q(z)$ are the same with $p_{g}(f(z))$ and $q_{g}(f(z))$ respectively, $p_{f}(w)$ and $q_{g}(w)$ being the functions corresponding to $g(w)$.

Theorem 3. If $w=f(z)$ and its inverse $z=g(w)$ are pseudo-regular, then $q_{f}(z)$ and $q_{g}(w)$ (accordingly $p_{f}(z)$ and $p_{g}(w)$ ) are equal in corresponding points.

Next we prove a theorem on pseudo-conformal representation, which is the generalization of Ahlfors" theorem(").

Theorem 4. Let $\Omega$ be a simply connected domain on $z(=x+i y)$-plane bounded by two segments $S_{1}$ and $S_{2}$ each on $R(z)=x_{1}$ and $x_{2}$ respectively, and by two curves $C_{1}$ and $C_{2}$ joining these segments, and let $T$ be a simply connected domain on $w(=u+i v)$-plane bounded by two segments $G_{1}$ and $G_{2}$ on $v= \pm \frac{a}{2}$ and two curves $L_{1}$ and $L_{2}$ joining these segments.

If $\Omega$ is represented pseudo-conformally on $T$ by $w=f(z) \equiv \xi(z)+i \eta(z)$ in such a way that $S_{1}, S_{2}, C_{1}$ and $C_{2}$ correspond to $L_{1}, L_{2}, G_{1}$ and $G_{2}$ respectively, then we have

(11) L. Ahlfors: loc. cit., $\left({ }^{3}\right)$. 


$$
a \int_{x_{1}}^{x_{2}} \frac{d x}{q(x) \theta(x)} \leqq \xi_{2}\left(x_{2}\right)-\xi_{1}\left(x_{1}\right)
$$

where

and

$$
\begin{aligned}
& \xi_{1}(x)=\min _{R(z)=x} \xi(z), \\
& \xi_{2}(x)=\max _{R(z)=x} \xi(z)\left({ }^{12}\right), \\
& \theta(x)=\text { length of the portion } \theta_{x} \text { of a straight line } R(z)=x \\
& \quad \text { cut by } C_{1} \text { and } C_{2}
\end{aligned}
$$

Proof.

$$
q(x)=\max _{1 R, z)=x} q(z) \text {. }
$$

The length $\gamma(x)$ of the image $\gamma$ in $T$ of $\theta_{x}$ satisfies

$$
\begin{aligned}
a^{2} \leqq \gamma(x)^{2} & =\left(\int_{\theta_{x}}\left|u_{y}+i v_{y}\right| d y\right)^{2} \\
& \leqq \theta(x) \int_{\theta_{x}}\left(u_{y}^{2}+v_{y}^{2}\right) d y \leqq \theta(x) \int_{\theta_{x}} q(x, y) J(x, y) d y \\
& \leqq q(x) \theta(x) \int_{\theta_{x}} J(x, y) d y
\end{aligned}
$$

Dividing the both sides by $q(x) \cdot \theta(x)$ and integrating with respect to $x$ from $x_{1}$ to $x_{2}$ we have

$$
a^{2} \int_{x_{1}}^{x_{2}} \frac{d x}{q(x) \theta(x)} \leqq \int_{x_{1}}^{x_{2}} \int_{\theta_{x}} J(x, y) d y d x
$$

the right hand side being the area of $T$,

$$
\leqq a\left(\xi_{2}\left(x_{2}\right)-\xi_{1}\left(x_{1}\right)\right) \text {. }
$$

\section{Remark.}

Taking the inequality

where

$$
a^{2}+\omega(x)^{2} \leqq \gamma(x)^{2}
$$

$$
\omega(x)=\xi_{2}(x)-\xi_{1}(x),
$$

we get after L. Ahlfors $\left({ }^{13}\right)$ a relation that:

If

$$
\int_{x_{1}}^{x_{2}} \frac{d x}{q(x) \theta(x)}>2
$$

then

$$
\xi_{1}\left(x_{2}\right)-\xi_{2}\left(x_{1}\right) \geqq a \int_{x_{1}}^{x_{2}} \frac{d x}{q(x) \theta(x)}-4 a .
$$

(12) We assume that $f(z)$ is continuous even on the boundary of $\Omega$.

(13) L. Ahlfors, loc. cit., $\left({ }^{3}\right)$. 
This inequality holds also even if the shape of $\Omega$ is more complicated as the case studied by Ahlfors ( ${ }^{1:}$ ).

Before we go to the generalization of Picard's theorem, we must define an isolated essential singularity of a psendo-regular function.

$z=a$ is called an isolated essential singularity of a pseudo-regular function $f(z)$, if, for a sufficiently small $\delta>0, f(z)$ is pseudo-regular for $0<\mid z$ $-a \mid<\delta$ and is discontinuous at $z=a$ in such a manner that there exist at least two sequences $\left\{z_{n}\right\}$ and $\left\{z_{n}\right\}$, each of which tends to $z=a$ as $n \rightarrow \infty$ and for which

$$
\lim _{n \rightarrow \infty} f\left(z_{\cdot}\right)=\alpha, \quad \lim _{n \rightarrow \infty} f\left(z_{n}{ }^{\prime}\right)=\beta, \quad \alpha \neq \beta
$$

$(f(z)$ may be not defined at $z=a)$.

Theorem 5. (Generalization of Picard's theorem) $\left({ }^{15}\right)$.

Let $f(z)$ be pseudo-regular for $0<|z|<1$ and let $z=0$ be its isolatod essential singularity. Then Picard's theorem holds if

$$
\lim _{\varepsilon \rightarrow 0} \int_{\varepsilon}^{t} \frac{d r}{r p(r)} \text { or } \lim _{\varepsilon \rightarrow 0} \int_{\varepsilon}^{1} \frac{d r}{r q(r)}\left({ }^{16}\right)
$$

diverges, where $p(r)$ and $q(r)$ denote the maximum of $p(z)$ and $q(z)$ on $|z|=r$ respectively.

In othei words, wnder this condition $f(z)$ takes every fnite value, except possibly at most one, infnitely many times in any neighbourhood of $z=0\left({ }^{17}\right)$.

Proof. It will bo sufficient if we prove the inconsistency of the assumption that $f(z)$ does not tak 0 and 1 in $0<|z| \leqq \delta, \delta>0\left({ }^{1 s}\right)$.

The domain $0<|z| \leqq \delta$ is transformed by $w=f(z)$ on a doubly connected Riemann surface $R_{w}$.

By the theorem of conformal representation, the interior of $R_{w}$ can be transformed by a regular function

$$
\zeta=F(w) \quad(w=\Phi(\zeta))
$$

(14) See L. Ahlfors, loc. cit., (").

(15) This result is the same with M. Lavrentieff's. H. Grötzsch proved it for the cise $q(z)<K$, and in $\$ 2$ and $\$ 3$ we use this theorem for this case.

(16) These two integrals diverge or converge simultaneously.

(17) This is a generalization of Picard's 2 nd theorem for a function which is homomorphic in $0<|z|<1$. In just the same manner pseudo-meromorphic functions can be defined and it will be obvious that Picard's theorem can be extended even for such functions under ana. logous conditions.

(15) Just as we do in the case of regular functions, we deal with $g(z)=\frac{f(z)-a}{b-a}$ instead of $f(z)$, if $f(z)$ takes $a$ and $b$, $a \neq b$, only finite times.

If $f(z)$ takes $a$ and $b$ only finite times, then for a suitably chosen $\delta>0, g(z)$ does not take 0 and 1 in $0<|z| \leq \delta$. 
on a domain

$$
r^{*}<|\zeta|<r, \quad 0 \leqq r^{*}, \quad r \leqq \infty .
$$

(Here we may assume that $|\zeta|=i$ corresponds to $\mid z=\delta$ ).

Then we have

$$
0<i^{*}, \quad i^{2}<\infty \text {. }
$$

For, if $\bar{r}^{*}=0$ or $\bar{r}=\infty$, then $\zeta=0$ or $\zeta=\infty$ must be an, isolated essential singularity, a pole or a regular point of $\Phi(\zeta)$.

Since $R_{w}$ has, however, no point on $w=0,1$ and $\propto, \zeta=0$ and $\zeta=\infty$ cannot be an isolated essential singularity (by the ordinary Picard's theorem for $\Phi(\zeta)$ ). On the other hand, if $\zeta=0$ is a regular point or a pole of $\Phi(\zeta)$, then the corresponding boundary of $R_{w}$ must be a single point, which is incompatible with the fact that there are at least two sequences $\left\{z_{n}\right\}$ and $\left\{z_{i}^{\prime}\right\}$, each tending to $z=0$, such that $f\left(z_{n}\right) \rightarrow \alpha$ and $\left.f^{\prime} z_{z}{ }^{\prime}\right) \rightarrow \beta, \alpha \neq \beta$; and in case of $\zeta=\infty$, we find aliso the assumption incompatible with the fact that $f(z)$ is pseudo-regular even on $|z|=\delta$.

Thus $0<|z|<\delta$ is transformed psoudo-conformally on

$$
0<\ddot{\eta}^{*}<|\zeta|<\ddot{q}<\infty
$$

by

$$
\zeta=F(f(z)) \equiv g(z)
$$

where $g(z)$ and $f(z)$ have the same $p(z)$ and $q(z)$ by Therem 1 .

If wo put

$$
s \equiv 2+i y=\log z, \quad \sigma \equiv \xi+i \eta=\log \zeta,
$$

then a domair.

$$
T: \quad \log r^{*}<\xi<\log \bar{r}, \quad|\eta|<\pi
$$

on $\sigma$-plane is transformed pseudo-conformally on a domain $\Omega$ on $s$-plane. (Taking the image $C$ of $\eta= \pm \pi$ in $0<|z|<\delta$, we cut this domain along $C$, and transform this by $s=\log z)$.

Put

$$
G(s) \equiv \log g\left(e^{s}\right) .
$$

As $q_{g}(s)$ is cqual to $q_{g}\left(e^{s}\right)$, the maximum $q(x)$ of $q_{s}(s)$ on $\theta_{x}(R(s)=x=\log r)$ is equal to the maximum of $q_{s}(z)$ on $|z|=r$, that is, equal to $q\left(r^{r}\right) \equiv q_{f}(r)$.

Hence we havo from Theorem $4(\theta(x) \equiv 2 \pi, a=2 \pi)$

$$
\int_{\log r_{1}}^{\log r_{2}} \frac{d x}{q(x)}=\int_{r_{1}}^{r_{2}} \frac{d r}{r q(r)} \leqq \xi_{2}\left(r_{2}\right)-\xi_{1}\left(r_{1}\right) \leqq \log r-\log r^{*}
$$

for any

$$
0<r_{1}<r_{3}<\delta
$$

If we let $r_{1} \rightarrow 0$, the integral on the left hand side becomes greater than any pre-assigned number, which is inconsistent becanse the right hand side is independent of $r_{!}$.

Thus we have arrived at contradiction.

\section{Corollary :}


$|z|<\infty$ cannot be transformed pseudo-conformally on $|w|<R<\infty$ by any pseudo-reguldir function for which

$$
\lim _{N \rightarrow \infty} \int^{N} \frac{d r}{r p(r)}=\infty \quad \text { or } \quad \lim _{N \rightarrow \infty} \int^{N} \frac{d r}{r q(r)}=\infty .
$$

In particular the theorem is true if $q(z)<K$ ( $K$ is a fixed constant).

\section{$\S 2$}

For the pseudoregular functions considered in $\S 1, u_{x}, u_{y}, v_{x}, v_{y}$ were continuous in all $D$. But Theorem 4 and 5 hold to be true even if, instead of being continuous all over $D, D$ can be divided into a finite or an enumerable number of sub-domains in which they are continuous. (Other conditions, especially that $u$ and $v$ are continuous in all $D$, must of course be still satisfied).

\section{Lemma.}

Let $D$ be a simply connected closed domain, and $D^{\prime}$ a closed sub-domain

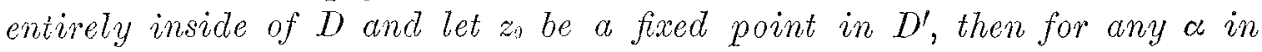
$D^{\prime}$, we can find two functions $f(z)$ and $g(z)$ both schticht and pseudo-regular in $D$ such that

$$
\left.\begin{array}{ccc}
f\left(z_{0}\right)=\alpha, & f(z) \equiv z & \text { on the boundary of } D, \\
g(\alpha)=z_{0}, & g(z) \equiv z & ,
\end{array}\right\}
$$

and for wihch

$$
q(z)<K\left(D, D^{\prime}\right)
$$

where $K\left(D, D^{\prime}\right)$ depends only on the configuration of $D$ and $D^{\prime}$.

Proof. It will be sufficient to prove only the existence of $f(z)$ for the following case :

$$
\begin{aligned}
& D: \quad|z| \leqq 1, \\
& D^{\prime}: \quad|z| \leqq \rho<1, \\
& \approx_{0}=0 \\
& q(z)<K(\rho) \leqq K\left(D, D^{\prime}\right),
\end{aligned}
$$

where $K(\rho)$ is a function of $\rho$. (Take as $g(z)$ the inverse of $f(z)$ ).

Put

$$
f(z)=\alpha(1-|z|)+z=\alpha(1-|z|)+|z| \cdot \frac{z}{|z|} \text {. }
$$

This is, as is easily seen, an automorphism of a unit circle in itself which satisfies (7).

As for (8), it is sufficient to prove this for the case when $\alpha$ is real.

If $\alpha$ is real, then

$$
\begin{aligned}
& u=\alpha\left(1-\sqrt{x^{2}+y^{3}}\right)+x, \\
& v=y,
\end{aligned}
$$


and

$$
\begin{array}{ll}
u_{x}=\frac{-\alpha x}{\sqrt{x^{2}+y^{2}}}+1, & u_{y}=\frac{-\alpha y}{\sqrt{x^{2}+y^{2}}}, \\
v_{x}=0, & v_{y}=1 .
\end{array}
$$

From this we have

$$
\begin{aligned}
p(z) & \equiv \frac{E+G}{J}=\frac{\left(2+\alpha^{2}\right) \sqrt{x^{2}+y^{3}}-2 \alpha x}{\sqrt{x^{2}+y^{2}-\alpha x}} \\
& =\frac{\left(2-\alpha^{2}\right) x^{2}+\left(2+\alpha^{2}\right) y^{2}+\alpha^{3} x \sqrt{x^{2}+y^{2}}}{\left(1-\alpha^{2}\right) x^{2}+y^{2}} \leqq \frac{2 x^{2}+3 y^{2}+|x| \sqrt{x^{2}+y^{2}}}{\left(1-\rho^{2}\right)\left(x^{2}+y^{2}\right)}
\end{aligned}
$$

As $|x| \leqq \sqrt{x^{2}+y^{2}}$ we have

$$
p(z) \leqq \frac{4}{1-p^{2}} .
$$

Although $u_{x}$ and $v_{*}$ each have a diccontinuity at $z=0$, we may, by the remark given at the beginning of $\$ 2$, consider this function in a class of pseudo-regular functions. ${ }^{19}$ )

Theorem 6.

Let $\sum$ be a simply connected infinitely many sheeted open Riemann surface on z-plane. Then the type of $\sum$ (hyperbolic or parabolic) is not altered if we deform $\sum$ topologically by a pseudo-regular function with bounded $q(z)$.

Here the deformation may be effected to only a small pant of $\sum$.

That is, the type of $\sum$ is not altered if we take a finite or an infinite number of mutually exclusive domains $D_{i}$ on $\Sigma$, and deform $\sum$ in each $D_{i}$ by a pseudo-regular function $f_{i}(z)$, which makes the boundary of $D_{i}$ unaltered and whose $q(z)$ is bounded uniformly in $i$. ( $D_{i}$ may have common projections on z-plane as it is sufficient that they are mutually exclusive considered on E.)

Proof. Evident from Theorm 5, Cor. 1.

According to this theorem, if we have any simply connected infinitely many sheeted opon Riemann surface whose type is already known, we can make an infinito number of Riemann surfaces of the same type by deform. ing $\sum$ by a pseudo-regular function with bounded $q(z)$.

For cxample, taking the Riemann surface $\Sigma$ of a modular function, which has an infinite number of logarithmic singularities on 0,1 and $\infty$, we have a class of hyperbolic Riemann surfaces by deforming $\sum$ in each neighbourhood of its logarithmic singularity, translating each singularity in an arbitrary direction by a suitably chosen length.

(19) We can also give a function which satisfies the conditions of lemma and whose four first partial derivatives are continuous. But such an example will be conplicated. 
This problem was first considered by A. Spoiser $\left({ }^{2 n}\right)$, and L. Ahlfors $\left({ }^{\circ 1}\right)$ solved this problem for the case that there exist three mutualy exclusive domains $E_{1}, E_{2}$ and $E_{3}$ each containing 0,1 and $\infty$ respectively, and that the deformation is effected in each $E_{i}(i=1,2,3)$.

Although L. Ahlfors' theorem says much more than this theorem, but his method cannot be applied if there is no such domain as $E$ and if, for example, the translation of singularities is effectod in such a manner that two singularities whose projections were at first different (for example 0 and ! respectively) have, after deformation, the same projections on $z$-plane, that is, if, rogarding only to the projections of singularities, the singularities corresponding to 0,1 or $\infty$ are confused by the translation.

We shall now deal with Riemann surfaces which have algebraic singularities. I. Ahlfors $\left({ }^{2}\right)$ proved the theorem:

Let $\sum$ be parabolic and let there be five mutually exclusive domains $D_{i}$ bounded by $C_{i}(i=1,2, \ldots, 5)$ respectively, then at least one of the section ("Insel") of $\sum$ by $D_{i}$ is one-sheeted (schlicht).

In other words:

Considering $\sum$ on Riemann's sphere and taking fire mutually exclusive domains $D_{i}$ bounded by $C_{i}(i=1,2, \ldots, 5)$, if each section ("Insel") $D_{i, p}\left({ }^{23}\right)(i=1,2, \ldots, 5 ; p=1,2, \ldots)$ of $\sum$ is at least two sheeted, then $\sum$ is hyperbolic.

If, except for a finite number of $D_{i, p}$, there is at least one point $z_{i, p}$ in each $D_{i, p}$, where all the sheets of $D_{l, p}$ have singularities (that is the case if all $D_{i, n}$ be two sheeted), and if the projection of $z_{i, p}$ is in $D_{i}^{\prime}$, which is entirely inside of $D$, then we can prove this theorem by our method.

We have only to take one point $z_{i}$ in each $D_{i}^{\prime}$ and to deform each $D_{i, p}$ by a psendo-regular function considered in the lemma such that it makes the boundary of $D_{i, p}$ unaltered, and translate $z_{i, p}$ into $z_{i, p}^{*}$ whose projection is $z_{i}$.

By Theorem 6 the Riemann surface $\Sigma^{\prime}$ thus obtained is of the same type with $\sum$, and $\Sigma^{\prime}$ is hyperbolic by the well-known theorem of Carathéodory-Bloch.

Added in proof (5 April, 1937)

While this paper was in the press, Nevanlinna's book "Eindeutige analytische Funktion" has appeared. In Chap. XII and XIII of this book. the type-problem of Riemann surfaces is treated and especially in Chap,

(20) A. Speiser, loc. cit. ( $\left.{ }^{6}\right)(a)$.

( $\left.{ }^{21}\right)$ L. Ahifors, loc. cit. ( $\left.{ }^{6}\right)$ (b).

$\left({ }^{\circ}\right)$ L. Ahlfors: loc. cit. ( ${ }^{(6)}$ (b). Here he proved this for six domains. The case of five domains is proved in $\left({ }^{6}\right),(d)$, see also $\left({ }^{6}\right),(c)$.

(23) $D_{i, p}$ corresconts to $D_{i}$. 
XIII \&8 pseudo-conformal representations are discussed under the name of "Abbildungen von beschränkter Exzentrizität." Here it is proved that most of the theorems on the conformal representation concerning the type problem are also valid for "Abbildungen von beschränkter Exzentrizität." It is easy to see that

$$
q(z)=\frac{1}{\sqrt{1-e^{2}(z)}}, p(z)=\sqrt{1-e^{2}(z)+} \frac{1}{\sqrt{1-e^{2}(z)}} .
$$

This concept of "Abbildungen ron beschänkter Exzentrizität" comes from L. Ahlfors $\left({ }^{24}\right)$.

\section{$\S 3$.}

In this chapter we deal with the class of Riemann surfaces $\sum$ whose singularities are all logarithmic and lie only on $1, e^{\frac{2 \pi}{3} i}$ and $e^{\frac{4 \pi}{3} i}$.

Such a class of Riemann surfaces was considered by R. Nevanlinna( $\left.{ }^{25}\right)$, A. Speiser $\left({ }^{96}\right)$, E. Ullrich $\left({ }^{27}\right)$ and Z. Kobayashi $\left({ }^{98}\right)$, and many conditions that $\sum$ is parabolic are obtained. Here we sha!l give a sufficient condition for hyperbolicity.

These surfaces are characterized by its "topological tree" introduced by A. Speiser $\left({ }^{59}\right)$, and almost all the conditions for parabolicity are given by the order of increment of "tree-function" $\mu(\theta)$.

If the increment of $\mu(\theta)$ is sufficiently small then $\Sigma$ is parabolic, and the greater the increment of $\mu(\theta)$ becomes, the more the surface $\sum$ becomes likely to be hyperbolic. But wo can show by an example that it is impossible to determine the type of surfaces of this class only by the order of increment of $\mu(\theta)$.

This goes as follows:

We consider a Riemann surface of this class which is symmetric and whose "tree-function" is of the following form:

$$
\begin{aligned}
& \mu(0)=1, \\
& \mu(\theta)=3 \cdot 2^{k}, \quad \pi n_{k}<\theta \leqq \pi n_{k+1}
\end{aligned}
$$

where $n_{k}(k=0,1,2, \ldots)$ are integers and

$$
0=n_{0}<n_{1}<n_{:}<\ldots<n_{k}<\ldots .
$$

The topological tree of $\Sigma$ is obtained if we take the topological tree of

$\left({ }^{4}\right)$ L. Ahlfor: loc. cit., ( $\left.{ }^{6}\right)(e)$. Here the notation "quasi-konforme Abbildungen" is used. It is to be remarked, that when I obtained the result of the present paper, the paper" of Ahlfors (") (e) had not yet appeared.

$(\because 5)$ R. Nevanlinna, loc. cit., ( $\left.{ }^{5}\right)$, (b).

$\left({ }^{26}\right)$ A. Speiser, loc. cit., $(\tau),(b)$.

$\left.{ }^{27}\right)$ E. Ullrich: Über ein Problem ron Herru Speiser, Comment. Math. Helv., 7 (1934).

("8) Z. Kobayashi, loc. cit., $\left({ }^{7}\right)$, (c) and $\left({ }^{8}\right)$, (a).

(9) A. Speiser: Über Riemannsche Flächen, Comment. Math. Helv., 2 (1930). 
Riemann surface of a modular function which is composed of segments of length $\pi$, and enlarge the segments of the $k$-th generation( $\left.{ }^{3 n}\right)$ of it to the length $\pi\left(n_{k}-n_{k-1}\right)$.

Theorem 7. If

$$
\frac{n_{k}}{n_{k+1}-n_{k}} \leqq K \text { and } \frac{n_{k+1}-n_{k}}{n_{k}-n_{k-1}}<K, \quad k \geqq 1
$$

for freed $K$, then $\sum$ is hyperbolic.

\section{Remark.}

If we put

$$
n_{k}=3 \cdot 2^{k-1}, \quad k \geqq 1,
$$

then (8) is satisfied for $K=2$, and the tree-function $\mu_{1}(\theta)$ of the Riemann surface $\sum_{1}$ corresponding to this $\left\{n_{k}\right\}$ is

$$
\begin{array}{rlrl}
\mu_{\mathrm{1}}(\theta) & =2 n_{k} \quad & \pi n_{k}<\theta \leqq \pi n_{k+1}, k \geqq 1, \\
& =3 & 0<\theta \leqq \pi n_{1}=3 \pi, \\
\mu_{\mathrm{t}}(0) & =1 . &
\end{array}
$$

On the other hand, for the Riemann surface $\sum_{a}$ of the inverse function of $w=e^{r^{z}}$, we have

$$
\begin{aligned}
& \mu_{2}(\theta)=2 n+3, \quad n \pi<\theta \leqq(n+1) \pi, \quad n=0,1,2, \ldots, \\
& \mu_{2}(0)=1,
\end{aligned}
$$

if we take suitably the "origin" of topological tree( $\left.{ }^{31}\right)$ of $\sum$.

Then

$$
\mu_{2}(\theta) \geqq \mu_{1}(\theta)
$$

for all $\theta \geqq 0$. And $\sum_{2}$ is paraboic, while $\sum_{1}$ is hyperbolic.

Proof of Theorem $\%$.

We deform $\sum$ into an easily manageable form as $Z$. Kobayashi( ${ }^{32}$ ) did.

Here the deformation may not always be partially conformal as in $Z$. Kobayashi's method, but it can also be pseudo-conformal under the condition that $q(z)$ is bounded.

Considering $\sum$ on w-plane, we first cut $\sum$ by a line running from origin to $\infty$ :

(39) We take a knot-point $P$ of topological tree $T$ as origin and call the segments which meet at $P$ "segments of 1st generation". After the segments of $(n-1)$-th generation have been determined, by "segments of $n$-th generation" we mean the segments which have common ends with those of $(n-1)$-th generation and which are not yet defined. See: A. Speiser, loe. cit., ( 5 ).

(31) We take as origin the knot point representing the sheet of Riemann surface which corresponds to the fundamental domain:

of s-plane.

$$
|\Pi(z)|<\pi, \quad\left|\pi\left(e^{z}\right)\right|<\pi
$$

(:a) Z. Kobayashi, loe cit. ( $\left.{ }^{8}\right)$, (a). 


$$
\operatorname{Arg} w=\frac{2}{3} p \pi, \quad p=0,1, \text { or } 2
$$

which goes over the logarithmic singularity of $\sum$, into an enumerable number of schlicht subdomains, each one of which is congruent to one of the following two domains

$$
\begin{array}{lll}
D_{1}: & -\frac{\pi}{3}<\operatorname{Arg} w<\frac{\pi}{3}, & 0<|w|<\infty ; \\
D_{:}: & -\frac{2}{3} \pi<\operatorname{Arg} w<\frac{2}{3} \pi, & 0<|w|<\infty .
\end{array}
$$

Cutting $D_{1}$ and $D_{2}$ by a bisecting line $\operatorname{Arg} w=0$ into two congruent parts, we transform each one of them thus obtained by a pseudo-regular function $W=W(w)$ :

or

$$
\begin{aligned}
& |W|=|w|, \quad \operatorname{Arg} W= \pm \frac{3}{2} \arg w \\
& |W|=|w|, \quad \operatorname{Arg} W= \pm \frac{3}{4} \arg w
\end{aligned}
$$

( + is taken for the upper part over $\operatorname{Arg} w=0$, and - is taken for the lower part under $\operatorname{Arg} w=0$ ) into a domain

$$
D: \quad 0<\operatorname{Arg} W<\frac{\pi}{2}, \quad 0<|W|<\infty .
$$

Here we notice that by this transformation $W=i$ corresponds to the logarithmic singularity of $\sum$, and that for this transformation $q(z)$ is bounded uniformly $\left(|q| \leqq \frac{3}{2}\right)$.

Again we transform $D$ by

$$
\zeta=\log \left(\frac{i-W}{i+W}\right)
$$

into a domain

$$
S: \quad-\infty<\xi<0, \quad 0<\eta<\pi
$$

of $\zeta(=\xi+i \eta)$-plane in such a manner that

$$
\begin{array}{llll}
\operatorname{Arg} W=0 & \text { corresponds to } & C_{1}: \xi=0,0<\eta<\pi, \\
\operatorname{Arg} W=\frac{\pi}{2}, 0<|W|<1 \quad, \quad & , & C_{2}: \eta=0,-\infty<\xi<0, \\
\operatorname{Arg} W=\frac{\pi}{2}, 1<|W|<\infty \quad, \quad & , & C_{3}: \eta=\pi,-\infty<\xi<0 .
\end{array}
$$

To $W=i$ corresponds $-\infty$ of $\zeta$-plane.

We take an enumcrable number of sheets of $S$ and connect them along 
their boundary lines in the same way as $D_{1}$ and $D_{\text {. }}$ are connected in $\Sigma$, so that we have in the end a surface $\Sigma^{\prime}$, which is essentially the same with the "cylindrical surface" of Z. Kobayashi, although its form is much simpler than that of Kobayashi's.

$\sum$ can be constructed in the following way:

Taking a topological tree $T$ of $\sum$, which is composed of segments of length $\pi$, affix to each segments $s$ of $T$ two sheets of $S$ along the

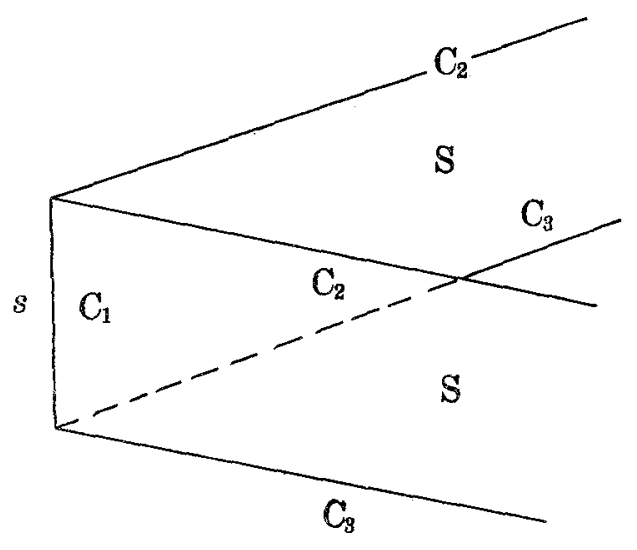

Fig. 1.

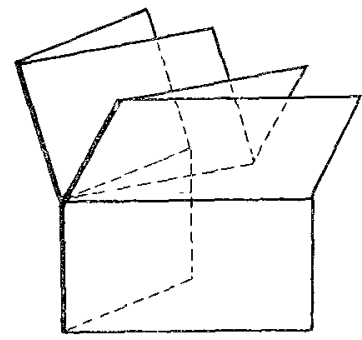

Fig. 2. boundary line $C_{1}$ of $S$ (Fig. 1).

Then identify the boundary line $C_{2}$ (or $C_{3}$ ) of two consecutive sheets of $S$, which are facing each other (to face means that they have the same end point on $T$ and that this point is on the same side of $T$ ) (Fig. 2.)

Thus we have an open surface $\Sigma$ ' which has no boundary in the finite part.

Then we fold $\Sigma^{\prime}$ in such a way that all the segments of $T$ which are at the same distance from the "origin" of $T$ come on the same place and consider it fixed on a $\zeta(=\xi+i \eta)$-plane such that $\Sigma$ lies in a quadrant $\xi>0$, $\eta>0$ (Fig. 3).

As is easily seen, $\sum^{\prime}$ has $2 \cdot 3 \cdot 2^{k} \equiv$ $2 \cdot \mu(\eta)$ sheets for $\pi n_{k}<\eta<\pi n_{k+1}$ (to each segment of $T$ correspond two sheets of $S$ ).

We cut $\Sigma$ by a straight line

$$
L_{k}: \quad \xi+\eta=\pi n_{k}, \quad k=1,2, \ldots,
$$

and transform the part $T_{k}(k=1,2, \ldots)$ of $\Sigma^{\prime}$ which is between $L_{k}$ and $L_{k+1}$

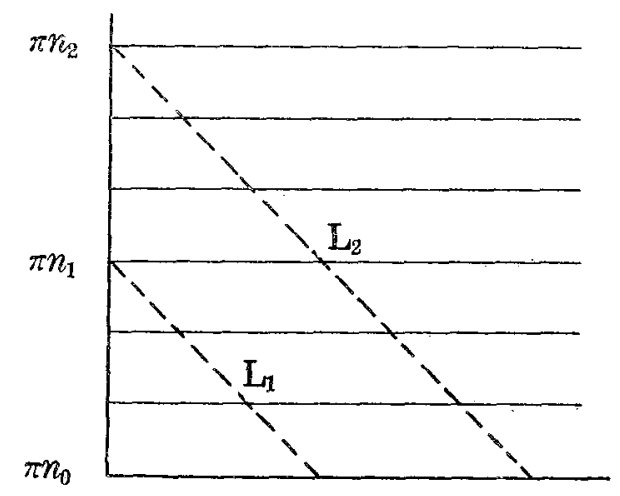

Fis. 3. into a rectangle $Q_{k}$ on $S(=\sigma+i \tau)$ plane which is between $\tau=0$ and $\tau=2 \pi$ (the length of one side of $Q_{k}$ is $2 \pi$ that of the other is $l_{k}$ which will be determined in the following).

This transformation is defined as follows: 
$T_{k}(k \geqq 1)$ consists of two classes of domains of $T_{k}^{\prime}$ and $T_{k}^{\prime \prime}\left(3 \cdot 2^{*}\right.$ sheets each).

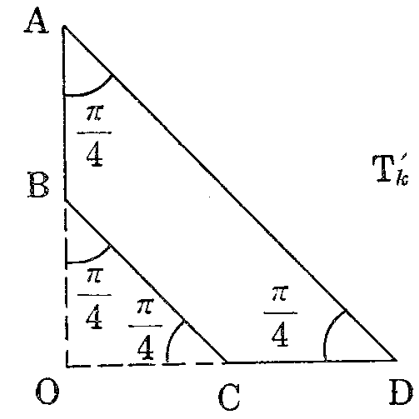

$$
\begin{aligned}
& \mathrm{AB}=\mathrm{CD}=\pi\left(n_{k+1}-n_{k}\right) \\
& \mathrm{OB}=\mathrm{OC}=\pi\left(n_{k}-n_{j}\right), j<k
\end{aligned}
$$

Fig. 4.

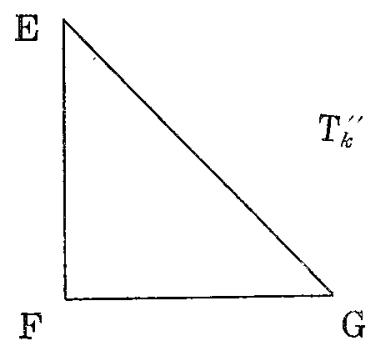

$$
\begin{aligned}
\mathrm{EF} & =\mathrm{FG} \\
& =\pi\left(n_{k+1}-n_{k}\right)
\end{aligned}
$$

Fig. 5 .

$T_{k}^{\prime}$ and $T_{k}^{\prime \prime}$ are connected in $T$ along the segments $\mathrm{AB}$ and EF, and two consccutive $T_{k}^{\prime}$ or $T_{k}^{\prime \prime}$ are connected along CD or FG respectirely.

We transform $T_{k}^{\prime}$ and $T_{k}^{\prime \prime}$ by pseudo-regular functions into domains $Q_{k}^{\prime}$ and $Q_{k}^{\prime \prime}$ (or into the domains $\bar{Q}_{k}^{\prime}$ and $\bar{Q}_{k}^{\prime \prime}$ which are symmetric to them) in such a way that $\mathrm{A}, \mathrm{B}, \mathrm{C}, \mathrm{D}$ of $T_{k}^{\prime}$ and $\mathrm{E}, \mathrm{F}, \mathrm{G}$ of $T_{k}^{\prime \prime}$ correspond to $\mathrm{A}^{\prime}, \mathrm{B}^{\prime}, \mathrm{C}^{\prime}, \mathrm{D}^{\prime}$ of $\Omega_{k}^{\prime}$ and $\mathrm{E}^{\prime}, \mathrm{F}^{\prime}, \mathrm{G}^{\prime}$ of $\Omega_{k}^{\prime \prime}$ respectively, and that this transformation is linear in each side of $T_{k}^{\prime}$ and $T_{k}^{\prime \prime}$.

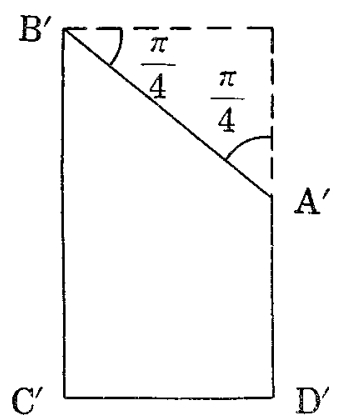

$\mathrm{B}^{\prime} \mathrm{C}^{\prime}=\alpha_{k}$

$\mathrm{C}^{\prime} \mathrm{D}^{\prime}=\frac{\alpha_{k}}{2}$

$\mathrm{A}^{\prime} \mathrm{D}^{\prime}=\frac{\alpha_{k}}{2}$

Fig. 6 .

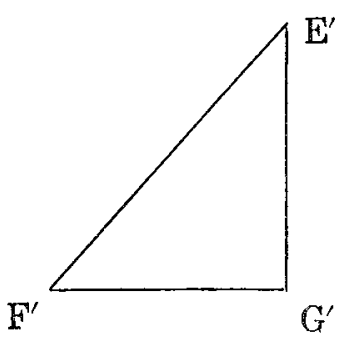

$\Omega_{l i}^{\prime \prime}$

$$
\mathrm{F}^{\prime} \mathrm{G}^{\prime}=\mathrm{E}^{\prime} \mathrm{G}^{\prime}=\frac{\alpha_{\mathrm{k}}}{2}
$$

Fig. 7 .

Then we connect $\Omega_{k}^{\prime}, \Omega_{k}^{\prime \prime}, \bar{\Omega}_{k}^{\prime}$ and $\bar{\Omega}_{k}^{\prime \prime}$ in the same way as the corres- 
ponding $T_{k}{ }^{\prime}$ and $T_{k}^{\prime \prime}$ are connected in $T_{k}$, and thus we construct a rectangle $Q_{k}$ out of $Q_{k}^{\prime}, Q_{k}^{\prime \prime}, \bar{Q}_{k}{ }^{\prime}$ and $\bar{\Omega}_{k}^{\prime \prime}$ (Fig. 8).

If we put $\alpha_{k}=\frac{2 \pi}{3 \cdot 2^{k}}$ then the length of one side of $Q_{k}$ is $2 \pi$ and that of the other is $l_{k}=\frac{\alpha_{k}}{2}=\frac{\pi}{3 \cdot 2^{k}}$

The transformation from $T_{k}^{\prime}$ or $T_{k}^{\prime \prime}$ to $Q_{k}^{\prime}, Q_{k}^{\prime \prime}, \bar{\Omega}_{k}^{\prime}$ or $\bar{\Omega}_{c_{c}}^{\prime \prime}$ is pseudo-regular and we shall calculate the maximal value of $q$ for each of them. For the sake of simplicity we put

$$
\begin{aligned}
& a=\frac{\pi}{\sqrt{2}}\left(n_{k}-n_{j}\right), \quad b=\frac{\pi}{\sqrt{2}}\left(n_{k+1}-n_{k}\right) \\
& \alpha=\alpha_{k}
\end{aligned}
$$

and consider $T_{k}{ }^{\prime}, T_{k}^{\prime \prime}$ and $\Omega_{k}{ }^{\prime}, \Omega_{k}{ }^{\prime \prime}$ suitably placed in $(x, y)$ and $(u, v)$-plane respectively.

I) From $T_{k}^{\prime}$ to $\Omega_{k}^{\prime}$ (Fig. 9):

$$
\begin{aligned}
& u=\frac{\alpha}{2 b} x, \\
& v=\frac{\alpha}{2}\left(1+\frac{y}{a+x}\right)\left(1-\frac{x}{2 b}\right) .
\end{aligned}
$$

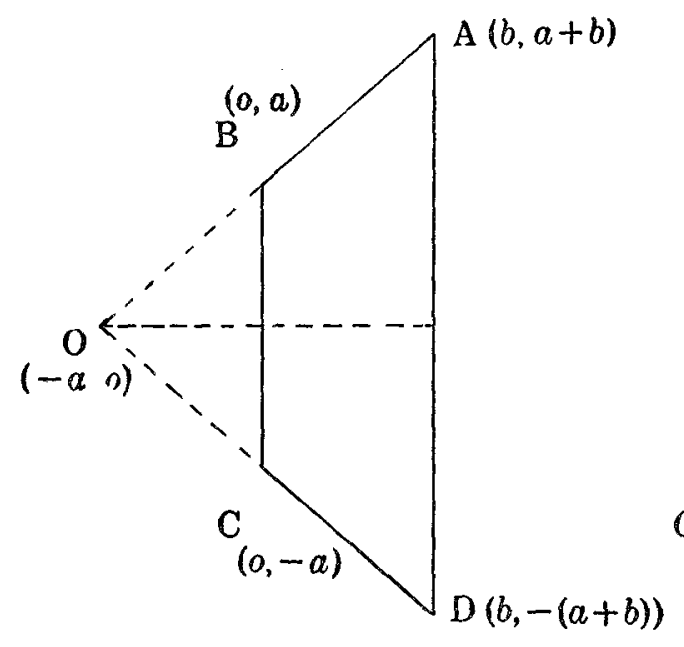

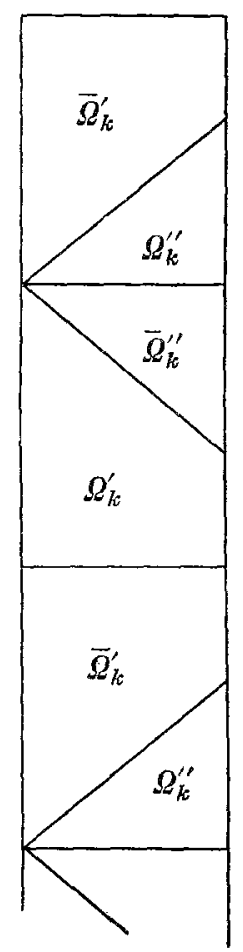

Fig. 8.

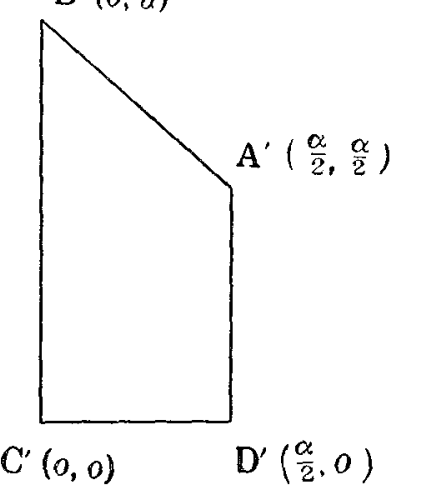

$(x, y)$-plane

Fig. 9.

$$
(u, v) \text { - plane }
$$

In this case

$$
p=q+\frac{\mathrm{I}}{q}
$$




$$
\begin{aligned}
& =\frac{\frac{1}{b^{2}}+\left\{\frac{y}{(a+x)^{2}}\left(1-\frac{x}{2 b}\right)+\frac{1}{2 b}\left(1+\frac{y}{a+x}\right)\right\}^{2}+\left\{\frac{1}{a+x}\left(1-\frac{x}{2 b}\right)\right\}^{2}}{\frac{1}{b(a+x)}\left(1-\frac{x}{2 b}\right)} \\
& =\left\{\frac{a+x}{b}+\frac{a+x}{4 b}\left(1+\frac{y}{a+x}\right)^{2}\right\}\left(1-\frac{x}{2 b}\right)^{-1} \\
& +\left\{\frac{b y^{2}}{(a+x)^{3}}+\frac{b}{a+x}\right\}\left(1-\frac{x}{2 b}\right)+\frac{y}{a+x}\left(1+\frac{y}{a+x}\right)
\end{aligned}
$$

and since $0 \leqq x \leqq b$ and $|y| \leqq a+x$ we have

$$
\leqq 4 \frac{a+b}{b}+2 \frac{b}{a}+2=6+4 \frac{a}{b}+2 \frac{b}{a} .
$$

2) From $T_{k}^{\prime \prime}$ to $\Omega_{k}^{\prime \prime}$ (Fig. 10):

$$
u=\frac{\alpha}{2 b} x, \quad v=\frac{\alpha(x+y)}{4 b} .
$$

In this case

$$
p=q+\frac{1}{q}=3 .
$$

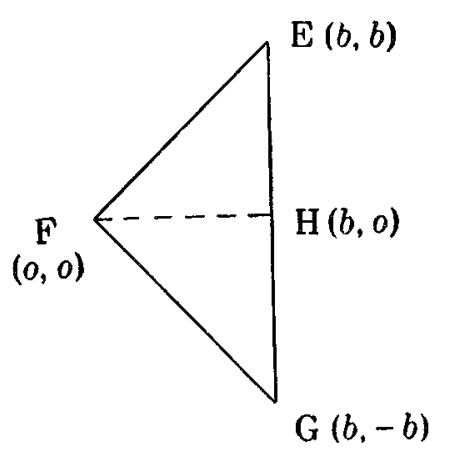

$$
\mathrm{EH}=\mathrm{FH}=\mathrm{GH}=b
$$$$
(x, y)-\text { plane }
$$

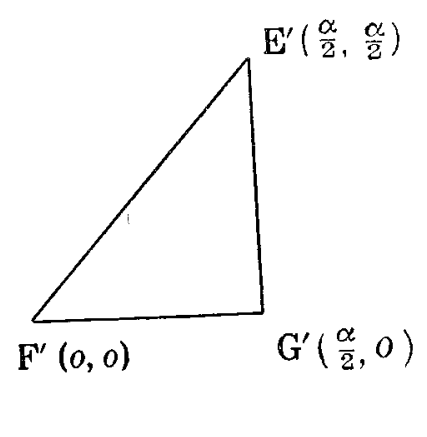

$E^{\prime} G^{\prime}=F^{\prime} G^{\prime}=\frac{\alpha}{2}$

Fig. 10.

We have always from 1) and 2)

and, since by asaumption

$$
p=q+\frac{1}{q} \leqq 6+4 \frac{a}{b}+2 \frac{b}{a}
$$

$$
\begin{aligned}
& \frac{a}{b}=\frac{n_{k}-n_{j}}{n_{k+1}-n_{k}} \leqq \frac{n_{k}}{n_{k+1}-n_{k}} \leqq K, \\
& \frac{b}{a}=\frac{n_{k+1}-n_{k}}{n_{k}-n_{j}} \leqq \frac{n_{k+1}-n_{k}}{n_{k}-n_{k-1}} \leqq K
\end{aligned}
$$


for all $k$, we have

$$
p=q+\frac{1}{q} \leqq 6(1+K)
$$

Performing these transformations for each $k(k \geqq 1)$, we connect the domains $\Omega_{k}$ thus obtained as is shown in Fig. 11.

If we determine the transformation of $T_{0}$ suitably so as to fill the part $Q_{v}(0<\tau<2 \pi$, $-\infty<\sigma<\sigma_{1}$, where $\sigma=$ $\sigma_{1}$ corresponds to $L_{1}$ in the transformation of $T_{1}$ on s-plane. See Fig. 11.), then we finally have the pseudoconformal transforma-

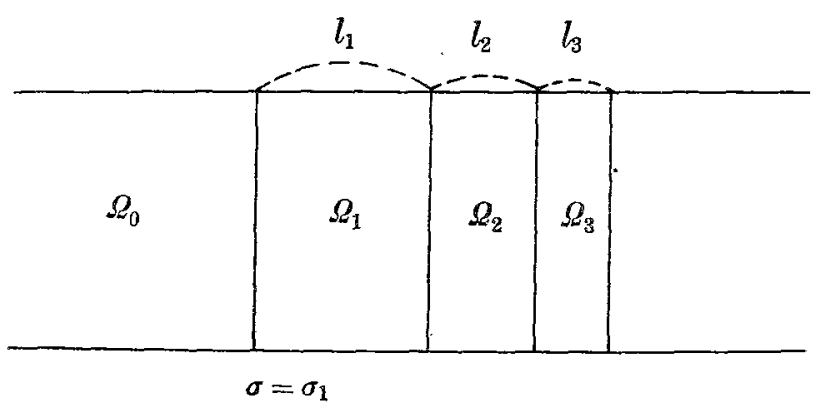
gFi. 11 . tion of all $\sum$ into a domain $\Omega=\Omega_{0}+\Omega_{1}+Q_{2}+\ldots$ of $s\left(=\sigma+i_{T}\right)$-plane which is between $\tau=0$ and $\tau=2 \pi$. (Since the transformations from $T_{k}^{i}$ or $T_{k}^{\prime \prime}$ to $Q_{k}{ }^{\prime}$ or $Q_{k}{ }^{\prime \prime}$ are all linear on the boundary, the transformation $\varphi$ from $\Sigma^{\prime}$ into $Q$ is continuous. Of course, we must choose the transformation of $T_{0}$ to $\Omega_{0}$ so as to make $\varphi$ continuous on $L_{1}$ )

$Q$ is bounded on the right side, because

$$
\sum_{k=1}^{\infty} l_{k}=\sum_{k=1}^{\infty} \frac{\alpha_{k}}{2}=\frac{1}{2} \sum_{k=1}^{\infty} \frac{2 \pi}{3 \cdot 2^{k}}=\frac{\pi}{3} .
$$

Thus we have a pseudo-conformal transformation $\varphi$ of $\Sigma^{\prime}$ in a domain $Q$ bounded on the right. As $p=q+\frac{1}{q}$ is uniformly bounded we have from Theorem 4, Cor. 1. that $\Sigma^{\prime}$ and accordingly $\sum$ is hyperbolic.

Added by the proof (23. March, 1937)

During the proof many publications concerning the Type-problem have appeared, under which the papers directly related with mine are as follows:

(1) C. Blanc, Le type des surfaces de Riemann simplement connexes, C. R. 202 (1936), p. 623.

(2) P. J. Myrberg, Uber die Bestimmung des Typus einer Riemannschen Fläche, Ann. Acad. Scient. Fennicae, Ser. A. XIV, No. 3.

In these papers the results of $\xi 3$ of my paper are obtained, but the way to get them are different.

Mathematical Institute, Osaka Imperia! University. 